\title{
EXPERIMENTAL ASSESSMENT OF THE QUANERGY M8 LIDAR SENSOR
}

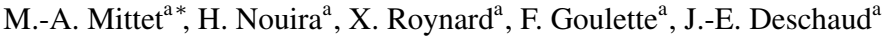

${ }^{a}$ MINES ParisTech, PSL Research University, Centre for robotics, 60 Bd St Michel 75006 Paris, France (marie-anne.mittet, houssem.nouira, xavier.roynard, franois.goulette, jean-emmanuel.deschaud)@ mines-paristech.fr

\section{Commission V, WG V/3}

KEY WORDS: Quanergy M8 / LIDAR / range imaging / calibration / point cloud / assessment

\begin{abstract}
:
In this paper, some experiments with the Quanergy M8 scanning LIDAR system are related. The distance measurement obtained with the Quanergy M8 can be influenced by different factors. Moreover, measurement errors can originate from different sources. The environment in which the measurements are performed has an influence (temperature, light, humidity, etc.). Errors can also arise from the system itself. Then, it is necessary to determine the influence of these parameters on the quality of the distance measurements. For this purpose different studies are presented and analyzed. First, we studied the temporal stability of the sensor by analyzing observations during time. Secondly, the assessment of the distance measurement quality has been conducted. The aim of this step is to detect systematic errors in measurements regarding the range. Differents series of measurements have been conducted : at different range and in diffrent conditions (indoor and outdoor). Finally, we studied the consistency between the differents beam of the LIDAR.
\end{abstract}

\section{INTRODUCTION}

Multi-laser scanning systems based on Time-of-flight (TOF) are widely used in engineering fields such as robot navigation, automatic guided vehicle (Moosmann and Stiller, 2011), and threedimensional measurement (Neumann et al., 2014) application in several industries. Several sensors of this kind are now available on the market. They have many advantages. Indeed, they provide 3D informations about their environment in real time, whitout contact, at wide range, and with high precision measurement (Amann et al., 2001).

There are two main approaches employed in Time-of-flight technology : direct and indirect TOF methods. The direct method is based on the measurement of time interval between an emitted and detected signals, which are very short pulsed lights (Kilpela et al., 2001). In the case of the indirect method, the distance is obtained by using a phase shift of a modulated light with either a sinusoidal signal or a pulsed signal (Jang et al., 2013).

The targeted applications require a high level of accuracy, so it is important to consider the distance range, the resolution and the accuracy of the sensor. To achieve this level of precision, it is required to study and analyzed the noise level of the laser scanner. Measurement made by multi-laser scanning systems are often distorded due to a set of phenomena. The measurements error of LIDAR system may come from different sources. (Reshetyuk, 2009) classify them into four groups : instrumental errors, errors related to the scanned object, environmental errors and methodological errors. Instrumental errors are due to the system design and can be removed by calibration or by design improvement. The environnement during the acquisition also has an influence, like for instance phenomena such as temperature, brightnss or humidity. Similarly, the characteristics (albedo, reflectivity, etc.) of the observed object has an influence. However, errors can occur according to the distance-to-object, or according to the indice angle. It is essential to determine the influence of these parameters on the quality of the distance measurement.
A well known system is the Velodyne HDL-64E S2. In the context of the autonomous vehicle of new sensors are emerging. An attractive cheaper model of LIDAR is the M8 LIDAR system. This is a new rotating LIDAR system funded by the Quanergy company. This unit consist in 8 lasers located on a spinning head. The aim of this paper is to provide a presentation of the M8 LIDAR system and its functionalities. In a second part some tests are carried out in order to characterize the sensor. Errors due to the environment such as errors due to the system itself will be pointed.

\section{QUANERGY M8 SENSOR PRESENTATION}

The Quanergy M8 LIDAR system consist in 8 2D line scanners located on a spinning head wich can spin at a rate from $5 \mathrm{~Hz}$ to $30 \mathrm{~Hz}$. The 8 lasers are spread out over a $20^{\circ}$ vertical field of view (FOV) and the entire unit rotates to give a full $360^{\circ}$ by $20^{\circ} \mathrm{FOV}$. An overview of the specifications for the M8 scanner is given in 1, and a picture of the scanner head is given in Figure 1. To enable the use of the sensor, Quanergy provide a SDK and an application to record data, both based on the so called framework ROS.

\begin{tabular}{|l|l|}
\hline Parameter & Specification \\
\hline Sensors & 8 lasers \\
Measurement technique & Time of Flight (TOF) \\
Measurement range & $150 \mathrm{~m}(80 \%$ reflectivity) \\
Range accuracy $(1 \sigma$ at $50 \mathrm{~m})$ & $<5 \mathrm{~cm}$ \\
Frame rate (Update frequency) & $10-30 \mathrm{~Hz}$ \\
Angular resolution & $0.03^{\circ}-0.2^{\circ}$ \\
Field of view & $\mathrm{H}: 360^{\circ}, \mathrm{V}: 20^{\circ}\left(+3^{\circ} /-17^{\circ}\right)$ \\
Laser class & 1 \\
Wavelenght & $905 \mathrm{~nm}$ \\
\hline
\end{tabular}

Table 1: Datasheet provided by Quanergy

\footnotetext{
${ }^{*}$ Corresponding author
} 


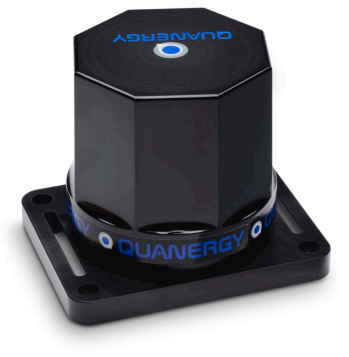

Figure 1: Quanergy M8 sensor

\section{SENSOR CHARACTERIZATION}

As mentionned by (Reshetyuk, 2009), measurement errors can have different sources. In this part, we attempt to evaluate the errors due to the system design and the environment. Indeed, it is necessary to determine the influence of these parameters on the distance measurement. Firstly, the repeatability of the measurement is presented. In a second time the consistency of the sensor is studied and finally the pre-heating time of the sensor was assessed.

\subsection{Measurement repetability}

Considering a static scene, the M8 is positioning in front of a planar wall, at a distance of approximatively $8.5 \mathrm{~m}$. Histograms of the distance measurement performed from a single laser point with almost $0^{\circ}$ vertical angle and $0^{\circ}$ horizontal angle were realized.

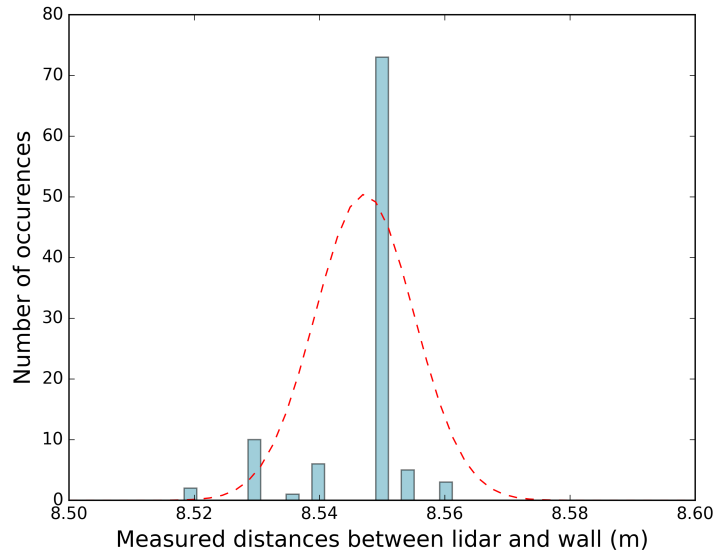

Figure 2: Histogram of 100 distance measurements performed for a single laser point (vertical angle : $0^{\circ}$, horizontal angle : $0^{\circ}$ )

On the Figure 2 the histogramm obtained for 100 successive measurement is presented. During this measurement the standard deviation is about $0.79 \mathrm{~cm}$ and the maximal difference between any two measurements is $3.9 \mathrm{~cm}$. The absence of measurement for some distances makes us think that a quantization phenomenon exists.

\subsection{Laser consistency}

To examine the consistency between the different laser of the M8, a point cloud of a wall in a room was acquired (Figure 3). Points measured are colored regards to their laser. Ideally, if the sensor had lower noise, the walls should have looked much thinner.

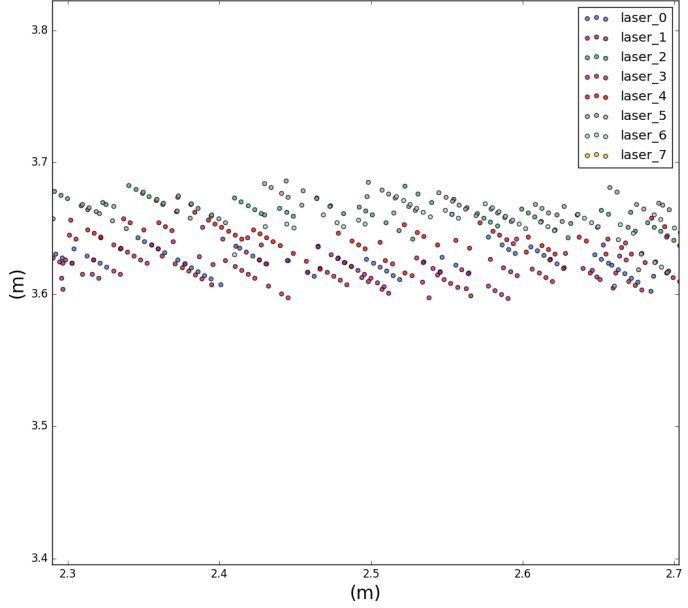

Figure 3: Top view of a wall

\subsection{Pre heating time}

We attempt to study pre-heating time for the M8 LIDAR system. The unit was set up to scan a wall at a known distance. In order to evaluate the influence of the environment, the manipulation was performed indoors and outdoors.

3.3.1 Indoor pre heating time In order to evaluate the pre heating time indoor, ranges were sampled every second for a period of appromatively one hour. On the Figure 4 each measurement is plotted as blue circles and the red line higlihts the average trend. The variability appears bounded approximativel by $\pm 15 \mathrm{~cm}$. We can observe a slight increase in the measured distance, with a stabilization at around 40 minutes.

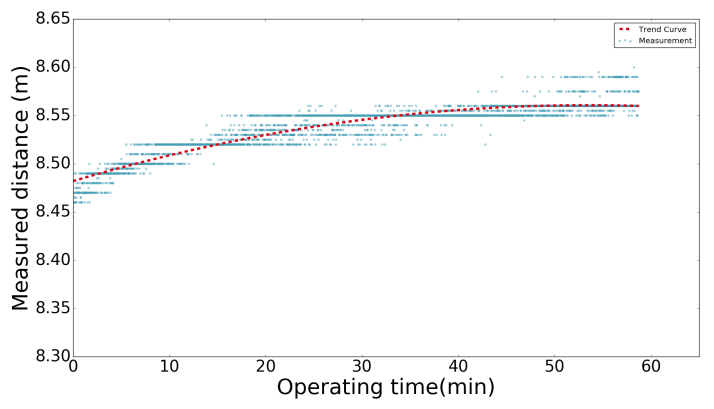

Figure 4: Pre heating time indoor

3.3.2 Outdoor pre heating time The same experiment was conducted outdoors, but over a period of 40 minutes.

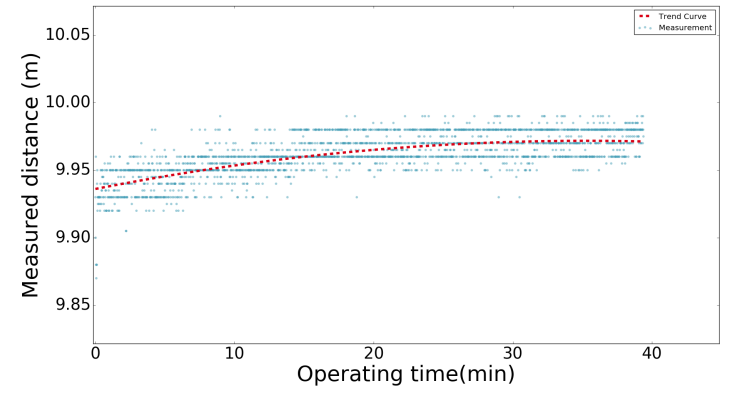

Figure 5: Pre heating time outdoor 
As mentionned in (Glennie and Lichti, 2011), a longer term study would be conducted. However, it is important to note that a too long pre-heating time is incompatible with many intended applications. We can observe that the pre-heating time seems consistent with the handling carried out indoor. Moreover, we can notice that the measurements appear to be more noisy than during the first manipulation.

3.3.3 Quantization On the Figure 6, we can observe a part of the measurement. The quantization phenoma is confirmed and the intervals appear to be arround $0,5 \mathrm{~cm}$.

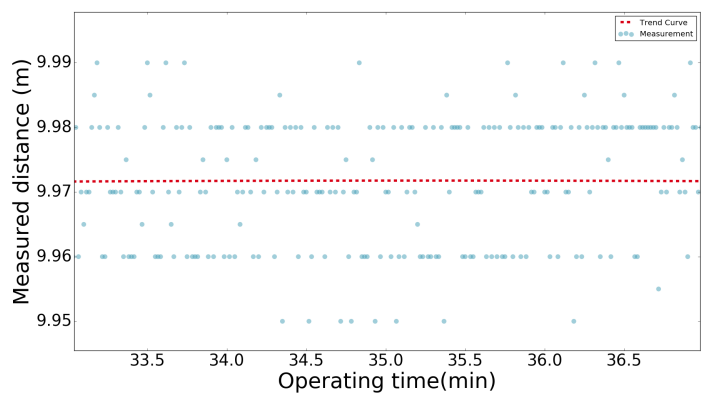

Figure 6: Illustration of the quantization phenomenon

\section{ACCURACY OF DISTANCE MEASUREMENT}

The M8 sensor is announced to be a smart sensing solutions for real-time 3D mapping and object detection, tracking, and classification. All these applications require the use of very accurate sensors. The time-of-flight sensors often suffer from depth-related deformation. It is necessary to assess these errors. A common way to evaluate these errors consist in positionning the sensor parallel to a wall at different known distances. The M8 LIDAR system is intended to be use both indoor and outdoor, so our study was conducted indoor and outdoor (Figure 7).

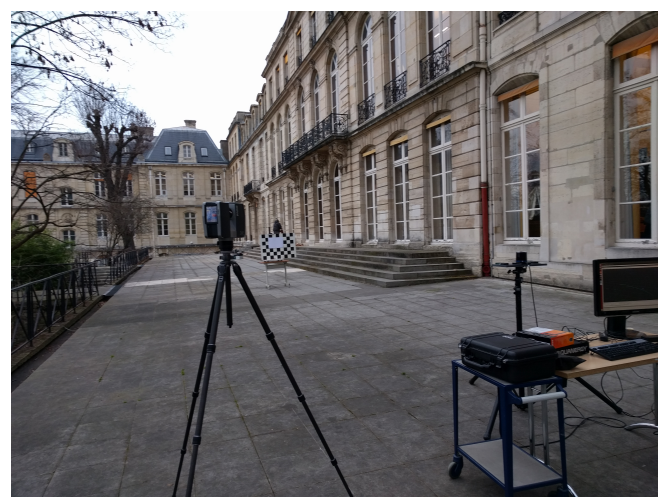

Figure 7: Experience on the evaluation of the accuracy of the distance measurement (outdoor)

To ensure the parallelism of the sensor during acquisitions, a fixation was realized. This fixation also permits to monitoring the true distance between the planar surface and the sensor. This sytem is composed consists of two laser rangefinders (BOSCH PLR 50C). The precision rangefinders is sufficient for using them as reference measurements $( \pm 4.5 \mathrm{~mm}$ at $50 \mathrm{~m})$. The fixation was performed using a computer numerical control milling machine whose accuracy is sub centimetric (Figure 8). Quanergy have given us precisely the position of the origin of the sensor. This one is in the center of the sensor, so we aligned the origin of the rangefinders with the origin of the sensor (Figure 8). The manufacturer also specifies that the angular step at 180 degrees is aligned with the cable, the zero being the opposite.

During the indoor acquisition a wall was used as surface. For the outdoor experiment a calibration pattern was used. The albedo can have a influence on the distance measurement, so the calibration pattern was covered (Figure 7). Both used surfaces were surveyed with a scanner laser (FARO Focus 3D) in order to assess the planarity with a device of assumed higher accuracy as the investigated sensor. The scanner also allowed us to control the truth distance between the surfaces and the sensor. Indeed a target was placed on the sensor. The position of the target is obtained thanks to acquisitions made by the scanner. So we used two controls to get the truth distance. The difference in distance measurement between the two controls is always less than $2 \mathrm{~mm}$.

Two acquisitions were made. First an indoor dataset, for distances ranging from $2 \mathrm{~m}$ to $14 \mathrm{~m}$, data were recorded every 2 meters. The second acquisition was performed outdoor between $10 \mathrm{~m}$ and $40 \mathrm{~m}$. First of all, every meter until $15 \mathrm{~m}$, and after that every $5 \mathrm{~m}$.
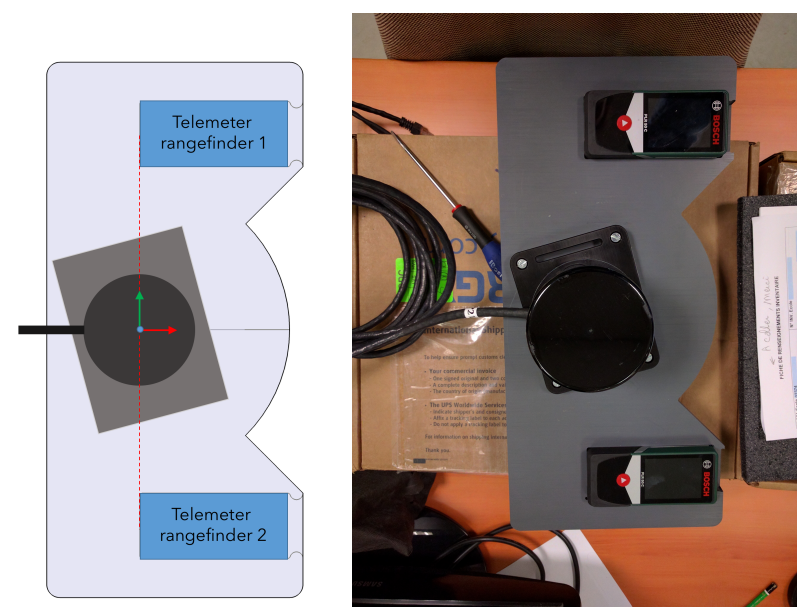

Figure 8: Fixation made for experimentation

\subsection{Results}

The aim of this experiment was to establish the deviation between real and measured distances as a function of the depth. To evaluate the sensor behavior under different conditions, two experiments were conducted: indoor, and outdoor. The results obtained are presented in Figure 9 and Figure 10.

The fixing realized has allowed to control the parallelism of the sensor relative to a planar surface. Thus, the true distance used is the point having a vertical and horizontal angle of $0^{\circ}$. In order to limit the noise, 100 successive measurements were used. The measurements were performed taking into account the previously determined pre heating time. These measurements are represented by crosses, while the red dots represent average distances, finally standard deviations are shown in blue.

\subsection{Indoor study}

Regarding the indoor acquisition, the error seems to be relatively constant. As depicted on the Figure 9, distortions of the central point vary from $-1.79 \mathrm{~cm}$ to $-4.47 \mathrm{~cm}$. The standard deviations calculated over the 100 point cloud acquired is fearly constant, 
except for one measurement. We observe that the measured distance is always too high compared to the real distance. Anyway, knowing the curve parameters allows the correction of the measured distances.

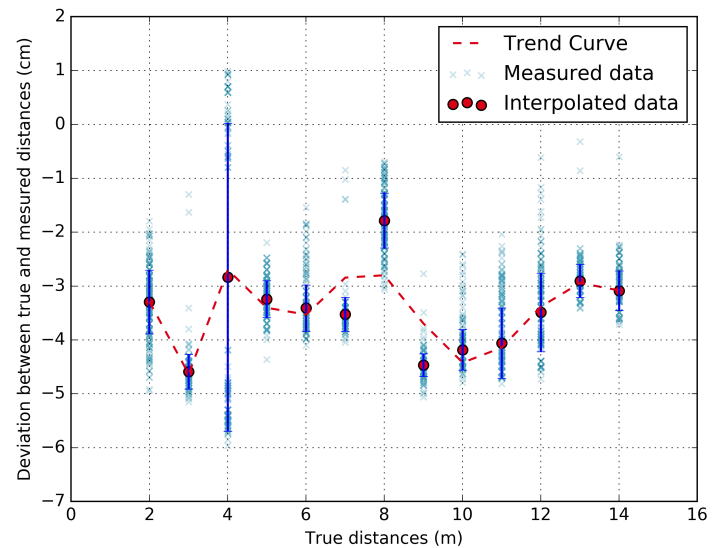

Figure 9: Deviations of measured distances with respect to true distances (indoor)

\subsection{Outdoor study}

As regards the outdoor acquisition, firstly the error seams to increase. However, stabilization of this error is observed from 23 meters. The distorsions of the central point vary from $1.70 \mathrm{~cm}$ and $16.03 \mathrm{~cm}$. In a logical way, the standard deviation increases with the distance.

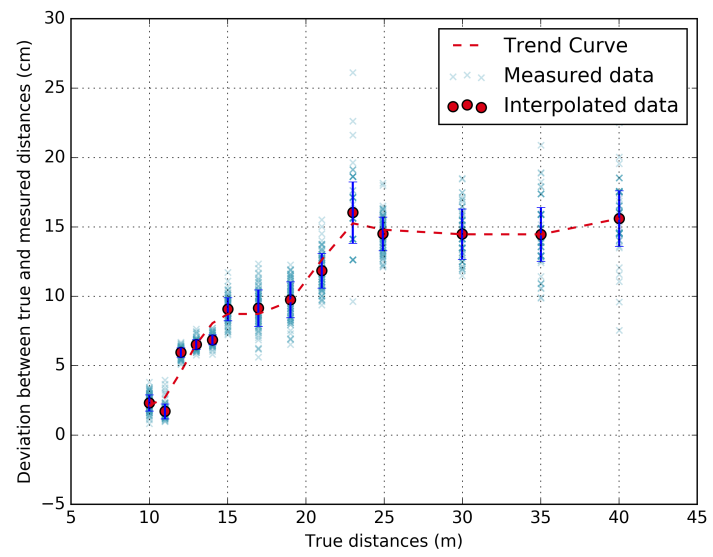

Figure 10: Deviations of measured distances with respect to true distances (outdoor)

The indoor and outdoor measurements have relatively different results (Figure 9, Figure 10). Indeed, on the common range between 10 meters and 14 meters, for the indoor acquisition, distortions of the central point vary from $-2.91 \mathrm{~cm}$ and $-4.19 \mathrm{~cm}$, while for the outdoor acquisition, distortions vary from $1.70 \mathrm{~cm}$ and $6,86 \mathrm{~cm}$.

According to the datasheet, the range accuracy expected for this sensor is $<5 \mathrm{~cm}(1 \sigma$ at $50 \mathrm{~m})$. However, for outdoor measurements, this value is exceeded before 50 meter s. In fact, this error is reached at a distance of 11 meters.

\section{DISCUSSION AND FUTURE WORK}

The Quanergy LIDAR is a new type of sensor. It is presented as a sensor for many applications, such as mobile mapping or autonomous vehicles. To assess this sensor, different experiments were conducted. First of all, a study of the repeatability of the measurement was conducted. In this experiment, a quantization effect was identified. This effect was confirmed in the study of the temporal stability of the sensor. The experiment on temporal stability did not permit to determine a precise short-term heating time. However, a longer phenomenon is not excluded. It would be interesting to conduct this study for much longer periods. Nevertheless, a too long rise in temperature could be incompabible with the intended applications.

The various experiment, and the fixation made were used to evaluate the error of distance measurement according to the distance. It was found that the error announced by the manufacturer at $50 \mathrm{~m}$ is reached much faster in terms of distance. Moreover, it has been shown that indoor and outdoor expriment provide different resultas in term of measurement error. For applications such as autonomous vehicle, excessive distance error is not acceptable, given the safety issue. It is important to note that the received sensor is one of the first versions released. Thus, it is possible that most of the results obtained here will be fixed in subsequent versions.

\subsection{Future work}

To complete our current work, it would be interesting to conduct all of these experiments on all lasers sensor. Moreover, beyond the measurement error study, the next step of our work will be to determine the calibration parameters to correct these errors. It could be interessting to determine the origine of the identified error. For example, in determining the existence of horizontal and vertical offset. Finally, it is plannned to examine kinematic data obtained by placing the sensor on a mobile mapping platform equiped with a navigation grade GNSS/INS unit. It will be then possible to study the accuracy of the sensor for high accuracy mobile mapping applications.

\section{CONCLUSION}

The aim of this paper was to investigate through a set of tests the quality of the Quanergy M8 sensor. This sensor is a new lidar, cheaper, and which can be sold in bulk. Our study was designed to assess the measurements provided by it in order to determine if it was possible to use it in the applications covered by the manufacturer. Several tests highlighted errors related to environment but also errors related to the system itself. Based on the obtained results, the physical limitations of the device could be assessed. Again, it is important to remember that the received sensor is one of the very first delivered. We hope that future versions will correct errors encountered. Accordingly, the arrival of this sensor can be seen as an opening for wider distribution of this type of sensor. In addition, other manufacturers are challenged, and offer more and more LIDAR models.

\section{REFERENCES}

Amann, M. C., Bosch, T., Lescure, M., Myllyla, R. and Rioux, M., 2001. Laser ranging: a critical review of usual techniques for distance measurement. Optical engineering 40(1), pp. 10-19.

Glennie, C. and Lichti, D. D., 2011. Temporal stability of the Velodyne HDL-64E S2 scanner for high accuracy scanning applications. Remote Sensing 3(3), pp. 539-553. 
Jang, J., Hwnag, S. and Park, K., 2013. Design of Indirect Timeof-Flight Based Lidar for Precise Three-Dimensional Measurement Under Various Reflection Conditions. In: Proceedings of the 4th International Conference on Sensor Device Technologies and Applications Design, pp. 25-29.

Kilpela, A., Pennala, R. and Kostamovaara, J., 2001. Precise pulsed time-of-flight laser range finder for industrial distance measurements. Review of Scientific Instruments 72(4), pp. 2197 2202.

Moosmann, F. and Stiller, C., 2011. Velodyne SLAM. In: Proceedings of IEEE Intelligent Vehicles Symposium, pp. 393-398.

Neumann, T., Ferrein, A., Kallweit, S. and Scholl, I., 2014. Towards a Mobile Mapping Robot for Underground Mines. In: Proceedings of 2014 PRASA, RobMech and AfLaT Int. Joint Symposium, Cape Town, South Africa.

Reshetyuk, Y., 2009. Terrestrial laser scanning: error sources, self-calibration and direct georeferencing. 